\section{Insulin lässt nur Männer abnehmen}

Insulin wirkt im Kopf von Frauen und Männern unterschiedlich. Bei einer künstlichen Erhöhung des Insulinspiegels im Gehirn durch Anwendung von Insulin-Nasenspray nehmen nur Männer ab. Dieser Effekt sei vor allem durch Reduktion des Fettgewebes gegeben, berichtet Dr. Sebastian Schmid. Frauen nähmen hingegen eher zu, wobei das Mehr an Gewicht vor allem durch Einlagerung von Wasser erklärbar sei, so Schmid.

DGIM Jahrestagung 2011

\section{Millionen Mädchen abgetrieben}

In Indien hat die kulturelle Norm, die nach einem männlichen Stammhalter verlangt, offenbar immer noch starken Einfluss. So wurden laut einer Studie im letzten Jahrzehnt schätzungsweise 3,1-6 Mio. weibliche Feten selektiv abgetrieben. Die Forscher konnten zeigen, dass dies vermehrt bei der zweiten Schwangerschaft der Fall ist, wenn bei der ersten bereits ein Mädchen geboren wurde. Dabei trieben Frauen mit höherer Bildung und Wohlstand überdurchschnittlich oft ab.

Jha P et al, Lancet 2011, 377:1921

\section{Infektionsherd Schulorchester}

Forscher haben die Blasinstrumente eines Schulorchesters untersucht und fanden 442 unterschiedliche Bakterien, 58 Schimmel- und 19 Hefepilze. Viele Bakterien waren hoch resistent gegenüber den üblichen Antibiotika. Sechs der 13 Instrumente waren in der Woche vor der Untersuchung gespielt, sieben waren einen Monat nicht benutzt worden. Blasinstrumente sollten daher nach Gebrauch komplett gesäubert werden, da die Keime nicht nur am Mundstück zu finden waren. Glass TR et al, General Dentistry 2011, 59:100
Wohltat für die Hände Arme kreuzen trickst Schmerz aus
Das Überkreuzen der Arme mindert die Fähigkeit des Gehirns, sensorische Stimuli an den Händen exakt zu lokalisieren. Dieser Effekt lässt sich möglicherweise in der Schmerztherapie ausnutzen.

Ärzte vom University College London haben untersucht, wie sich die Haltung der Arme auf die Schmerzwahrnehmung auswirkt. Dafür verabreichten sie 20 Probanden schmerzhafte Laserpulse auf den Handrücken. In einer Versuchsanordnung legten die Studienteilnehmer ihre Arme und Hände seitlich vom Körper auf einen Tisch, in der anderen überkreuzten sie die Arme. Die Schmerzwahrnehmung wurde mithilfe einer numerischen Skala von 1-100 gemessen und zusätzlich mit dem EEG registriert. Das Ergebnis: Schmerzimpulse wurden als schwächer wahrgenommen, wenn die Probanden die Arme überkreuzt hielten.

Der Studienleiter begründet den Effekt so: Im Gehirn gebe es „Karten“ für die rechte Körperhälfte und für die Umgebung auf der rechten Seite des Körpers, die normalerweise gleichzeitig aktiviert würden. Wenn dies - beim Überkreuzen der Arme - nicht mehr passiert, würden bestimmte Stimuli weniger effektiv verarbeitet.

\section{Schmerzverarbeitung veråndert}

\section{Haben Biopsien bald ausgedient?}

\section{Prostatakrebs mit neuem Urintest auf der Spur}

Ein neuer Urin-Test auf Prostatakrebs könnte etlichen Männern mit erhöhten PSA-Werten unnötige Biopsien ersparen. Mit dem Urin-Test wird gezielt nach zwei Genen gesucht, die miteinander verschmolzen sind. Diese Gen-Anomalie ist pathologisch und kommt bei Prostatakrebs vor.

Findet man diese Gen-Anomalie, heißt das nahezu immer, dass der Betroffene Prostatakrebs hat. Und diese Gen-Anomalie ist jetzt mit dem neuen Test auch im Urin nachweisbar. In der aktuellen Studie hatte das Team um den Pathologen Dr. Scott Tomlins von der Univer- sität Michigan Urinproben von mehr als 1300 Männern mit erhöhten PSAWerten untersucht und dabei nach dem pathologischen Gen geforscht.

\section{Je höher, desto aggressiver}

Ergebnis: In der Gruppe mit dem höchsten Urintest-Score für das kranke Gen hatten 69\% der Männer ein histologisch nachgewiesenes Karzinom. In der Gruppe mit mittlerem Score waren es $43 \%$ und in der Gruppe mit geringem Score 21\%. Darüber hinaus stellten die Forscher fest, dass die Befunde des Urintests mit der Aggressivität des Tumors korrelierten.

(eb) 